19 Revue d'histoire du XIXe siècle

Société d'histoire de la révolution de 1848 et des

révolutions du XIXe siècle

13 | 1996

(Re)penser le XIXe siècle

\title{
Le mot du président
}

\section{OpenEdition}

Journals

Édition électronique

URL : http://journals.openedition.org/rh19/92

DOI : 10.4000/rh19.92

ISSN : $1777-5329$

\section{Éditeur}

La Société de 1848

\section{Édition imprimée}

Date de publication : 1 décembre 1996

ISSN : 1265-1354

Référence électronique

"Le mot du président », Revue d'histoire du XIXe siècle [En ligne], 13 | 1996, mis en ligne le 28 juin 2005 consulté le 22 septembre 2020. URL : http://journals.openedition.org/rh19/92 ; DOI : https://doi.org/ $10.4000 /$ rh19.92

Ce document a été généré automatiquement le 22 septembre 2020.

Tous droits réservés 


\section{Le mot du président}

1 Le conseil d'administration de la Société d'histoire de la révolution de 1848 et des révolutions du XIX ${ }^{e}$ siècle nouvellement élu lors de l'assemblée générale du 30 mars 1996 a démocratiquement modifié l'organigramme de notre société. Alain Corbin, président, et Jacqueline Lalouette, secrétaire générale, souhaitaient être remplacés dans leurs fonctions. Ont été élus secrétaire général, Frédéric Chauvaud - par ailleurs maître de conférences à l'Université de Poitiers -, et président, Jean-Luc Mayaud - professeur d'histoire contemporaine à l'Université Lumière-Lyon 2-. Les fonctions de trésorier que j'assurais ont été confiées, jusqu'à la prochaine assemblée générale, au trésorier adjoint Pierre Lenoël. Que soient ici, au nom de la société, chaleureusement remerciés Alain Corbin et Jacqueline Lalouette pour l'énergie et le temps qu'ils ont consacrés à notre toujours jeune association fondée il y a maintenant 92 ans. La seule énumération éditoriale des sept années du mandat d'Alain Corbin témoignent d'un très important bilan. La publication de sept livraisons de la revue 1848, révolutions et mutations au XIXe siècle devenue Revue d'histoire du XIXe siècle, révèle l'orientation donnée par le président et l'équipe rassemblée autour de lui. Les numéros à thème ont été systématisés et leur contenu montre l'élargissement des préoccupations de notre société qui embrasse dorénavant le XIX ${ }^{\mathrm{e}}$ siècle dans sa totalité : les Jeunesses, les Utopies, Le silence, L'exil et L'incendie précèdent cette dernière livraison qui, couronnant l'édifice, reprend les actes de la journée du 12 octobre consacrée à " Re-penser le XIXe siècle ". Ont également été organisés deux importants colloques en relation avec des associations partenaires et le Centre d'histoire du XIX ${ }^{e}$ siècle présidé par Alain Corbin : le colloque " Femmes dans la cité, 1815-1871 " tenu à Paris en novembre 1992 paraîtra sous peu aux éditions Créaphis; celui ayant pour thème " La barricade ", qui nous a réuni à Paris les 17-19 mai 1995 est en cours de fabrication aux Presses de la Sorbonne.

2 Si Alain Corbin ne préside plus notre société et si Jacqueline Lalouette n'en est plus secrétaire générale, il est évident que tous deux demeurent présents et que nous comptons tous sur leur participation assidue à nos activités. L'héritage sera maintenu et nous tenterons de le faire fructifier. Ce " nous" n'est pas de majesté: il est reconnaissance de l'équipe existante et renouvelée qui tente d'assurer le fonctionnement de la société et qui allège le rôle du président. En prévision des tâches qui nous attendent, le comité de rédaction de la Revue d'histoire du XIXe siècle a été 
renforcé : outre les membres du bureau de la société et les responsables permanents des rubriques - auxquels Pierre Lenoël a accepté de se joindre -, il a été décidé de confier la direction de chaque numéro à un ou deux rédacteurs occasionnels. C'est ainsi que le considérable travail de préparation de la revue devrait être quelque peu réduit pour ceux qui en assument bénévolement la charge. L'allégement est toutefois très relatif puisque dès cette année la Revue d'histoire du XIXe siècle devient semestrielle. L'aide financière apportée par le Centre national du livre et la réduction des coûts de fabrication par la modernisation de nos méthodes de travail - les pages de la revue sont livrées " prêtes à clicher " à l'imprimeur - autorisent ce progrès qui nous paraît nécessaire. Il n'est toutefois possible, rappelons-le, que par le dévouement et les compétences d'Évelyne Lejeune-Resnick, Frédéric Chauvaud, Jean-Jacques Yvorel et tous ceux qui participent à la préparation de notre revue.

L'orientation donnée à notre société et à sa revue sera donc conservée. Elle permet ainsi de préparer le cent-cinquantenaire de la révolution de 1848 sur de solides bases méthodologiques et scientifiques. Car notre association ne renie pas l'objet de sa fondation : l'appel à adhésion de février 1904 annonçait les buts de la Société d'histoire de la révolution de 1848 , précisant qu'il s'agissait "avant tout [d']organiser scientifiquement le labeur historique devenu possible sur une période défigurée par une foule de légendes [et] en seconde ligne, [de] travailler à répandre les idées de 1848, considérées comme le développement naturel et logique des idées de la Révolution ". Certes, aujourd'hui, les enjeux militants n'apparaissent plus de même nature, tant l'idée et la pratique républicaines semblent partagées par tous, ou presque tous. La reconnaissance d'utilité publique, le 14 avril 1933, avait entériné la décision de 1916 étendant la compétence de l'association à l'étude de "l'histoire de cette époque [1848] et en général à tous les mouvements politiques et sociaux à partir du XIX ${ }^{e}$ siècle " 1 . Était toujours affirmée sa vocation scientifique, vocation qui n'empêche nullement son implication entière dans la préparation du centenaire de $1848^{2}$ qui la mobilise et lui permet une vaillante renaissance après son interruption en 1940. Il nous semble donc devoir préparer le cent-cinquantenaire, tout en tentant de conserver notre identité et en affirmant notre présence scientifique sans verser dans le seul acte commémoratif.

4 Notre société organise ainsi un colloque international qui se tiendra les 22 et 23 février 1998 dans les locaux du Palais Bourbon. Il est pris en compte par la cellule mise en place à l'Assemblée nationale pour l'organisation d'une exposition itinérante sur le thème "1848, l'Europe des images. Les révolutions européennes et l'imagerie politique " et à laquelle nous participons, aux cotés de multiples chercheurs et universitaires européens dont beaucoup sont membres de la Société d'histoire de la révolution de 1848. Les commissaires généraux de l'exposition sont Ségolène Le Men (Paris XNanterre), Nicole Moulonguet (Assemblée nationale) et Philippe Kaenel ((Université de Lausanne). Cette exposition sera présentée au Palais Bourbon en janvier-février 1998, avant de gagner la Suisse - Musée national de Prangins -, l'Italie, puis Nuremberg... l'itinéraire définitif n'étant pas encore totalement arrêté.

5 Le colloque, placé sous l'autorité de Maurice Agulhon, est préparé par un groupe de pilotage constitué au sein de notre société et dirigé par son président. Quatre thèmes généraux sont retenus, qui occuperont chacun une demi-journée du colloque: le suffrage " universel "; la question sociale; provinces et colonies/Paris; Europe, le " printemps des peuples". Pour chacun des thèmes, il sera fait appel à des communications à la fois synthétiques et problématiques, reflets des préoccupations 
scientifiques de notre fin de $\mathrm{XX}^{\mathrm{e}}$ siècle. La dimension internationale du colloque implique également que nous ne nous enfermions pas dans une vision trop francofrançaise : seront sollicités un grand nombre d'intervenants étrangers.

6 Les actes du colloque seront bien entendu publiés. Mais l'activité éditoriale de notre société ne peut se limiter à ce seul volume. Les prochaines livraisons de la Revue d'histoire du XIXe siècle prépareront le cent-cinquantenaire. Le numéro 14, annoncé pour mai 1997 et coordonné par Francis Demier et Jean-Luc Mayaud, tentera un bilan historiographique des études sur 1848 réalisées depuis l'impulsion donnée par le centenaire dont l'histoire sera d'ailleurs retracée. Le numéro 15, publié en novembre 1997 et dirigé par Jean-Claude Caron et Michèle Riot-Sarcey, abordera les perspectives de recherche et les aspects méthodologiques. Le numéro 16, prévu pour mars 1998 pourra éventuellement contenir des articles plus spécifiques et des études de cas proposés par les membres de la société qui n'auraient pu trouver place dans les publications précédentes. Reste enfin que nous envisageons de publier rapidement un recueil d'articles fondateurs et difficilement trouvables concernant 1848 , tel le célèbre "1848-1830-1789, comment naissent les révolutions" d'Ernest Labrousse... Les prochains numéros de notre revue comporteront enfin une nouvelle rubrique réservée aux informations concernant les manifestations organisées pour le centcinquantenaire : le rôle de notre société est également de rendre compte et de tenter de coordonner les diverses initiatives françaises et étrangères. De tels projets impliquent la participation de chacun. Toute information peut être adressée à la société - à son siège social ou à l'adresse de son président -. Notre réseau national et international d'informateurs et de correspondants doit se renforcer: toutes les propositions sont bienvenues.

7 Le présent numéro publie donc les actes de la journée d'étude organisée par notre société le 12 octobre dernier à Paris. Chacun, parmi la centaine de présents de la salle Malher, a pu constater la qualité des interventions et le grand intérêt des débats. Incontestablement cette journée répondait à un besoin. Elle a été préparée par Michèle Riot-Sarcey, Rosemonde Sanson et Jean-Claude Caron. Nous devons les remercier doublement, pour n'avoir pas ménagé leur peine et pour avoir proposé à notre société l'organisation d'une telle manifestation : le succès de cette journée leur doit beaucoup. La publication d'actes de colloque moins de deux mois après son déroulement est exceptionnelle. La performance mérite en soi d'être soulignée. Remercions les auteurs des communications qui ont tous respecté le court délai qui leur avait été laissé pour remettre leur copie définitive. Mais, pour l'accomplissement de cet exploit, il a fallu tout le dévouement et toute la compétence des organisateurs de la journée auxquels se sont joints les rédacteurs des synthèses des tables rondes qui ont œuvré au plus vite: que Claude-Isabelle Brelot, Odile Krakovitch, Natalie Petiteau et Frédéric Chauvaud reçoivent ici l'expression de la gratitude de la société. Enfin, l'équipe entière de rédaction de la revue s'est totalement mobilisée : nos vifs remerciements s'adressent avec reconnaissance à Évelyne Lejeune-Resnick, à Jean-Jacques Yvorel et à MarieClaude Charles, chargée de " formater " les multiples fichiers informatiques. Souhaitons le meilleur accueil à ce numéro de la Revue d'histoire du XIXe siècle. Le succès de la journée du 12 octobre nous encourage à persévérer. De telles initiatives seront poursuivies, notamment pour la préparation du colloque de décembre 1997 " L'invention du XIX siècle ", organisé par la Société des études romantiques, le Musée d'Orsay et notre société, et dont le programme est en cours d'élaboration. 


\section{NOTES}

1.Voir, pour l'historique de notre société, les articles de Rémy GOSSEZ, "Éléments pour un historique de la Société d'histoire de la révolution de 1848 et des révolutions du XIX ${ }^{\mathrm{e}}$ siècle, 1904-1940 ", dans 1848, révolutions et mutations au XIX ${ }^{e}$ siècle, nº 1, 1985, pp. 17-38 et " La Société de "1848" (1945-1980) ", ibidem, n², 1986, pp. 13-22.

2.Sur cet épisode, voir Rémi GOSSEZ et Philippe VIGIER, avec Jean-Claude CARON et Pierre LENOËL, " 1848-1948, les printemps des peuples. Le centenaire en France ", dans Histoires de centenaires ou le devenir des révolutions. Contributions à l'histoire des centenaires des révolutions de 1830, 1848, 1870 et 1871 en France et en Europe-1848, révolutions et mutations au XIX ${ }^{e}$ siècle, $\mathrm{n}^{\circ}$ 5, 1989, pp. 45-53 et Thomas SNÉGAROFF, Le centenaire de la révolution de 1848 en France, Mémoire de maîtrise sous la direction de Jean-Yves Mollier, Université de Versailles/Saint-Quentin-en-Yvelines, 1996, 208 fº 